\title{
Electron beams produced by innovative photocathodes based on nanodiamond layers
}

\author{
L. Velardi $\odot,{ }^{1,2,,{ }^{*}}$ V. Turco, ${ }^{1}$ L. Monteduro, ${ }^{1}$ G. Cicala, ${ }^{2,3}$ A. Valentini,${ }^{3}$ and V. Nassisi ${ }^{1}$ \\ ${ }^{1}$ Laboratorio di Elettronica Applicata e Strumentazione LEAS, INFN, Department of Physics, \\ University of Salento-Lecce, Via Provinciale Lecce-Monteroni, 73100 Lecce, Italy \\ ${ }^{2}$ CNR-ISTP, Via Amendola 122/D, 70126 Bari, Italy \\ ${ }^{3}$ INFN-Sezione di Bari, Department of Physics, University of Bari, Via Orabona 4, 70126 Bari, Italy
}

(Received 21 December 2018; published 27 September 2019)

\begin{abstract}
The investigation of two different photocathodes (PCs) based on nanodiamond (ND) layers, irradiated by a $\mathrm{KrF}$ nanosecond excimer laser (wavelength, $\lambda=248 \mathrm{~nm}$; photon energy, $E_{\mathrm{Ph}}=5 \mathrm{eV}$ ) is reported. The ND layers were deposited by means of a pulsed spray technique. Specifically, the active layer of each PC consisted of untreated (as-received) and hydrogenated ND particles, $250 \mathrm{~nm}$ in size, sprayed on a $p$ doped silicon substrate. The ND-based photocathodes were tested in a vacuum chamber at $10^{-6} \mathrm{mbar}$ and compared to a Cu-based one, used as reference. All the photocathodes were irradiated at normal incidence. The quantum efficiency $(\mathrm{QE})$ of the photocathodes was assessed. QE values of the ND-based photocathodes were higher than that of the reference one. In particular, the hydrogenated ND-based PC exhibited the highest $\mathrm{QE}$ due to the negative electron affinity that results from the surface terminated by hydrogen. Additionally, the photocathode surface/local temperature and the multiphoton process contribution to the electron emission were studied.
\end{abstract}

DOI: 10.1103/PhysRevAccelBeams.22.093402

\section{INTRODUCTION}

The production of electron beams by photo and thermionic emission is becoming increasingly important and of growing interest in the scientific community. To date, the cathode represents a key component of electron injectors in synchrotrons, free electron lasers [1-3], linear accelerators (for example energy recovery linacs, ERLs) [4] and systems for imaging and diffraction. Many large-scale particle-accelerator facilities have been and are today employed in the development and characterization of cathodes, such as BNL [5-7], CERN [8], DESY [8] and SLAC [7,9]. In Italy, the National Institute of Nuclear Physics (INFN) is the biggest research organization involved in the design, production and characterization of cathodes for FLASH [3] and PITZ [9] facilities at DESY and for the SPARK_LAB [1,10-12] laboratory.

For applications, a cathode is required to be not only a good electron emitter (high quantum efficiency, QE), but also it must have a good lifetime/robustness, fast response time and low emittance. In general, photocathode (PC) technology is based on metals $(\mathrm{Cu}, \mathrm{Mg})[2,8,10,12-14]$ and

\footnotetext{
*luciano.velardi@le.infn.it

Published by the American Physical Society under the terms of the Creative Commons Attribution 4.0 International license. Further distribution of this work must maintain attribution to the author(s) and the published article's title, journal citation, and DOI.
}

semiconductors (CsI, GaAs, $\mathrm{Cs}_{3} \mathrm{Sb}, \mathrm{Cs}_{2} \mathrm{Te}, \mathrm{K}_{2} \mathrm{CsSb}, \mathrm{GaN}$, diamond, etc.) $[2,8,13,15-19]$. Metal photocathodes are characterized by low efficiency, good response time and are resistant to contamination. On the contrary, those based on semiconductors show high efficiency and slower response time but are sensitive to contamination.

A method used to reduce the PC work function is surface cesiation (i.e., covering the surface with cesium, Cs), which provides a negative electron affinity condition (NEA, i.e., the vacuum level shifted beneath of conduction band minimum) and therefore a photoemission enhancement. NEA photocathodes are bright electron sources and have high quantum efficiency because the electrons injected in the conduction band are emitted directly into the vacuum, without any energy barrier at the surface.

Wide band gap $(\geq 5 \mathrm{eV})$ semiconductors (e.g., AlN, BN, CsI, diamond, etc.) are an important class of materials because they match the energy of ultraviolet (UV) photons, making them very attractive solar blind UV detectors. In particular, diamond is a very interesting wide gap material of $5.47 \mathrm{eV}$ with a low electron affinity of $0.35-0.5 \mathrm{eV}$ [20] that becomes negative $(-1.27 \mathrm{eV})$ when its surface is hydrogenated [21,22]. Moreover, it has superior chemical-physical properties such as high chemical inertness, high mechanical hardness, high radiation hardness, good thermal conductivity and high secondary electron emission coefficient that allows it to work under harsh external conditions.

The production of diamond films is based on chemical vapor deposition (CVD) techniques, such as hot filament 
CVD (HFCVD) $[23,24]$ and microwave plasma enhanced CVD (MWPECVD) [25,26], working at high substrate temperatures $\left(\geq 500^{\circ} \mathrm{C}\right)$. These high temperatures are not compatible with many devices, for example microchannel plates where the photocathode is implemented directly on top of them to increase the QE. Usually, microchannel plates are made of glass and cannot be heated to temperatures higher than $350^{\circ} \mathrm{C}$.

In recent years, new techniques have been developed in diamond technology, allowing different possibilities in terms of deposition conditions and materials utilized as carbon sources. Besides CVD deposition techniques, which generally produce diamond films with excellent physical and chemical properties, starting from $\mathrm{CH}_{4}$ highly diluted in $\mathrm{H}_{2}$, another cheap and straightforward method is the pulsed spray technique $[27,28]$ which allows the deposition of layers from a dispersion of nanodiamond (ND) particles in water.

This last technique was used to produce high QE UV photocathodes based on nanodiamond layers $[18,19,29]$ in the vacuum ultraviolet range $(140-210 \mathrm{~nm})$. The QE was evaluated using a deuterium lamp coupled to a monochromator for the wavelength selection and a calibrated National Institute of Standards and Technology (NIST) Si photodiode.

In this paper, the photoemission characteristics of the ND-based photocathodes produced by pulsed spray technique were measured using a $\mathrm{KrF}$ excimer laser (monocromatic wavelength $\lambda=248 \mathrm{~nm}$ ). The $\mathrm{QE}$ was compared with that of a reference $\mathrm{Cu}$ photocathode. The local PC temperature (due to the laser-surface interaction) and the multiphoton absorption process were also investigated.

\section{MATERIALS AND METHODS}

A graphite rich nanodiamond powder [30] with an average grain size of $250 \mathrm{~nm}$ was utilized to fabricate the active layer of the photocathodes. The powder was dispersed in deionized water and sonicated for $30 \mathrm{~min}$ by a Bandelin Sonoplus HD2070 system and then sprayed on $p$ doped silicon substrates, $12 \times 12 \mathrm{~mm}^{2}$. The deposition temperature was set at $150^{\circ} \mathrm{C}$, which is a temperature much lower than those used in CVD techniques $\left(500-900^{\circ} \mathrm{C}\right)$. For additional details about the setup and the parameters used for the pulsed spray technique, see $[18,19]$.

Two types of devices were manufactured using untreated (as received, $\mathrm{ND}_{\mathrm{ar}}$ ) and hydrogenated (treated, H-ND) nanodiamond particles. The H-ND particles were obtained by treating the $\mathrm{ND}_{\mathrm{ar}}$ powder in $\mathrm{H}_{2}$ microwave plasma. The plasma was generated in a homemade cylindrical stainless steel ASTeX-type reactor. During the hydrogenation process, the working pressure, the microwave power, the $\mathrm{H}_{2}$ flow rate and the process time were held constant at $50 \mathrm{mbar}, 1250 \mathrm{~W}$, $200 \mathrm{sccm}$ and $1 \mathrm{~h}$, respectively. Details about the hydrogenation process can be found in [19].

\section{ELECTRON EMISSION FROM PHOTOCATHODES}

\section{A. Experimental apparatus for emission measurements}

Figure 1 shows a sketch of the experimental apparatus in which the laser-induced photoemitted charge measurements were performed. The apparatus includes a $\mathrm{KrF}$ excimer laser (photon energy of $5 \mathrm{eV}$ ) with pulse time duration $\tau$ of about $20 \mathrm{~ns}$ measured as full width at half maximum (FWHM) and a vacuum stainless-steel chamber equipped with a quartz window, a turbomolecular pump, capacitors, anode and a holder for photocathodes. The measurements were carried out setting the anode-cathode distance at $3 \mathrm{~mm}$ and applying an accelerating voltage up to $6 \mathrm{kV}$ which corresponds to an electric field of $2 \mathrm{MV} / \mathrm{m}$. The maximum value of $6 \mathrm{kV}$ was due to the occurrence of electrical breakdown in the accelerating gap responsible for plasma discharge. The $\mathrm{KrF}$ laser energy was varied in the range from $10^{-3}$ to $10^{-2} \mathrm{~J} / \mathrm{cm}^{2}$, higher values were not achieved because of the plasma formation.

The laser beam was divided by a beam splitter. One laser beam was then focused by a thin lens (100 cm focal length) producing a spot dimension of $40 \mathrm{~mm}^{2}$ on the photocathode. The other laser beam was sent to a fast Hamamatsu R1328U-02 photodiode in order to control the evolution time of the laser pulse, and to use it as trigger. The photocathodes were fixed on a holder connected to the chamber by an insulating flange (Fig. 1).

Four capacitors $(350 \mathrm{pF} / 40 \mathrm{kV})$ were connected between the anode and ground (chamber walls) in order to stabilize the applied voltage (which accelerates the electrons) and allow measurement of long pulses.

To analyze the beam current, it is necessary to consider the cathode holder together with the chamber walls as a transmission line. The value of characteristic impedance was calculated to be approximately $50 \Omega$. Therefore, to avoid signal reflections and to record the real current pulses, the cathode was connected to ground by 12 parallel

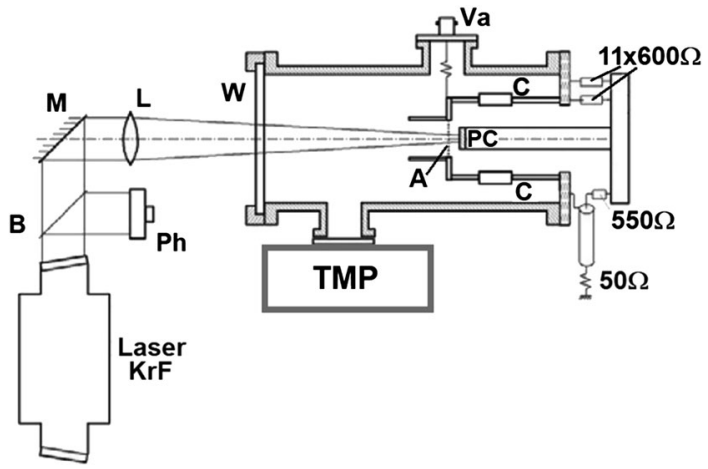

FIG. 1. Experimental apparatus: KrF, excimer laser; Ph, photodiode; $\mathbf{B}$, beam splitter; $\mathbf{M}$, mirror; $\mathbf{L}$, lens; $\mathbf{W}$, quartz window; TMP, turbomolecular pump; $\mathbf{C}$, capacitors; Va, anode voltage connector; A, anode; PC, photocathode. 


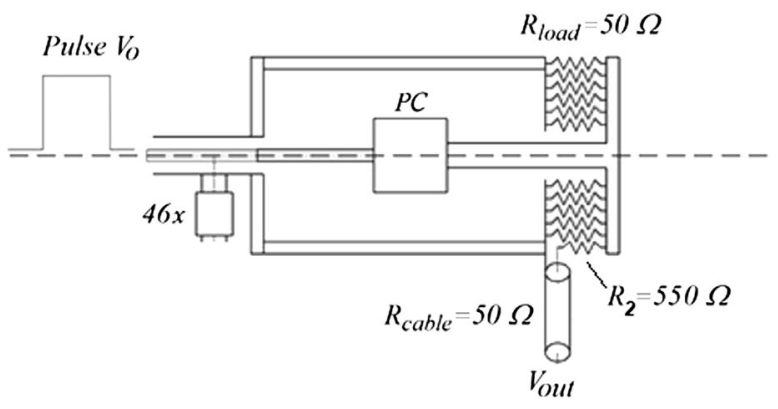

FIG. 2. Shunt calibration.

resistors of approximately $600 \Omega$ each, used as shunt with a total load resistance $\left(R_{\text {load }}\right)$ of $50 \Omega$. One of these, a $550 \Omega$ resistor $R_{2}$, was connected in series to a $50 \Omega$ coaxial cable $\left(R_{\text {cable }}\right)$, as shown in Fig. 2 . The $R_{\text {cable }}$ was used to transmit the current signal to the oscilloscope.

The shunt calibration was performed by simulating an electron beam and, for this purpose, a rectangular voltage pulse $V_{0}$ was applied to the cathode holder through a $46 \mathrm{x}$ attenuator to avoid the oscilloscope damage. It was generated by a fast switch with rise time of a few ns.

The resulting output voltage $V_{\text {out }}$ was

$$
V_{\text {out }}=\frac{50}{550+50} V_{o}
$$

The value of the current $i_{0}$ was

$$
i_{o}=\frac{V_{o}}{R_{\text {load }}}=\frac{550+50}{50} \frac{V_{\text {out }}}{R_{\text {load }}} .
$$

Figure 3 shows the calibration results. The temporal evolution of the input pulse $V_{0}$ is represented by the upper trace (yellow), whereas the $V_{\text {out }}$ signal is shown by the bottom trace (pink) with a rise time of 2.5 and $5 \mathrm{~ns}$, respectively. According to Eq. (1), $V_{\text {out }}$ is less than $V_{0}$ and the attenuation factor obtained by Eq. (2) was $0.247 \mathrm{~A} / \mathrm{V}$.

This calibration allows us to measure the photoelectric charge as a function of the voltage, for the QE calculation.

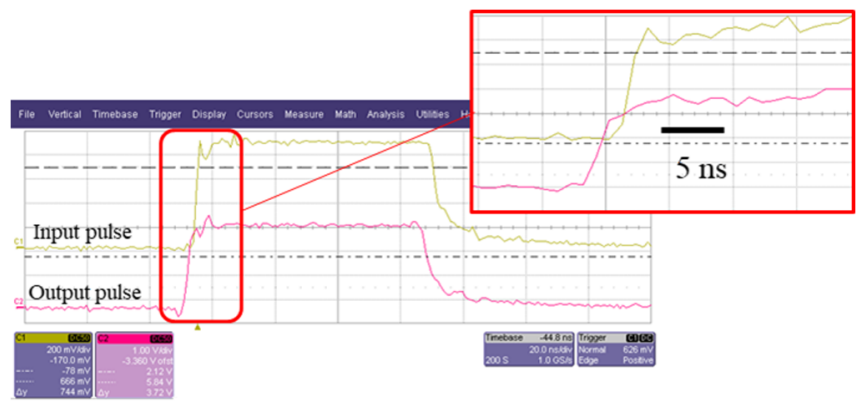

FIG. 3. Experimental results of the temporal response time measurement of apparatus. Yellow trace: input pulse $V_{0}$; pink trace: output signal $V_{\text {out }}$.
The anode was a stainless steel mesh with four grids per $\mathrm{mm}^{2}$. The anode grid passed approximately $60 \%$ of the incident laser light.

\section{B. Theory of photoemission}

The electron emission induced by laser-matter interaction is governed by the generalized Richardson equation [31]:

$$
J=J_{0}+J_{n-\text { photon }}
$$

where the term $J_{o}$ represents the contribution due to the thermionic mechanism. The current density $J_{n-\text { photon }}$ is related to the $n$-photon absorption processes, given by

$J_{n-\text { photon }}=\sum_{n=1}^{N+1} a_{n} I^{n}(t) A(1-R)^{n} T^{2}(t) f\left(\frac{n h \nu-\phi}{k T}\right)$,

where $a_{n}$ is the $n$th quantum coefficient related to the matrix element of the quantum $n$-photon process, $I$ is the incident laser irradiance, $A$ is the Richardson constant, $R$ is the PC optical reflection, $T$ is the temperature, $\phi$ the work function of the emitter, $k$ is the Boltzmann constant, $N$ is the largest integer less than $\varphi / h v, f\left(\frac{n h v-\varphi}{k T}\right)$ is the Fowler function with $n$ number of photons and $h \nu$ the photon energy.

Note that the $n$-photon absorption process for $n>1$ is relevant when the incident photon energy is less than the band gap.

\section{Temperature temporal profile}

In this section, it is shown that the thermionic component $J_{0}$ is negligible because the maximum increase in temperature of the cathode, due to the laser irradiation, is of the order of few Kelvin.

From Eq. (4), one can observe that the temporal behavior of $J$ is governed by the variables irradiance $I(t)$ and temperature $T(t)$ that depend on time. The temporal profile of the irradiance $I$ is easily deduced by the laser source, while the temporal shape $T$ on the photocathode surface is more complicated. Indeed, the temporal behavior of the temperature is described by the heat equation:

$$
\frac{\partial T(t, \vec{x})}{\partial t}=\nabla^{2} T(t, \vec{x})
$$

where $x$ is the depth from the surface.

The $T(t)$ solution is expressed in terms of the following integral [32-34],

$$
T(t)=T_{0}+C I_{0} \int_{0}^{t} g\left(t-t^{\prime}\right) t^{\prime-1 / 2} d t^{\prime},
$$

where $T_{o}$ is the initial temperature, $C$ is a constant dependent on the cathode material and $I_{o}$ is the laser irradiance. 


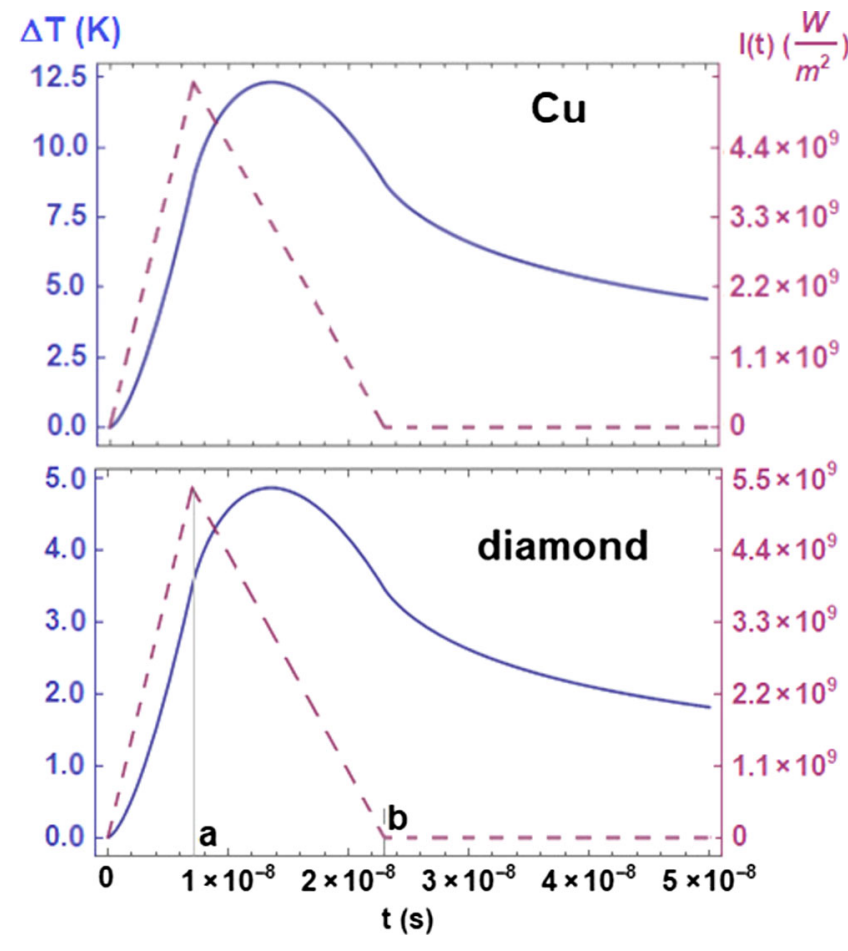

FIG. 4. Temporal evolution of the cathode surface temperature (blue trace) and the laser irradiance (dashed burgundy trace), approximated by a triangular piecewise function. $g(t)$ :

The triangular piecewise function is employed to define

$$
g(t)= \begin{cases}\frac{t}{a}, & 0 \leq t \leq a \\ \frac{(a+b-t)}{b}, & a \leq t \leq a+b \\ 0, & \text { otherwise, }\end{cases}
$$

although the real temporal profile of the laser pulse has a more complicated shape.

Figure 4 shows the $\Delta T$ variation of the $\mathrm{Cu}$ and diamond cathode surface (blue trace) and the laser pulse temporal profile (dashed burgundy trace) measured and calculated, respectively, at the maximum laser energy $(2.5 \mathrm{~mJ})$ employed in this work. The $a$ and $b$ parameters, shown in Fig. 4, govern the shape of the triangle. This approximation, however, apart from being good enough to this paper's aim, allows to obtain analytical well-known solutions of the heat equation (5) [34]. It is worth noting that the temperature value does not change significantly over the entire duration of the pulse. These results are in agreement with the assumption of constant temperature in Eq. (4). The higher $\Delta T$ variation found for the $\mathrm{Cu}$ surface is due to its specific heat capacity $[385 \mathrm{~J} /(\mathrm{kg} \cdot \mathrm{K})]$ lower than that of the diamond $[509 \mathrm{~J} /(\mathrm{Kg} \cdot \mathrm{K})][35]$.

\section{RESULTS AND DISCUSSION}

Figure 5 shows the extracted photocharge, measured in coulomb, of $\mathrm{Cu}, \mathrm{ND}_{\mathrm{ar}}$ and $\mathrm{H}-\mathrm{ND}$ photocathodes as a

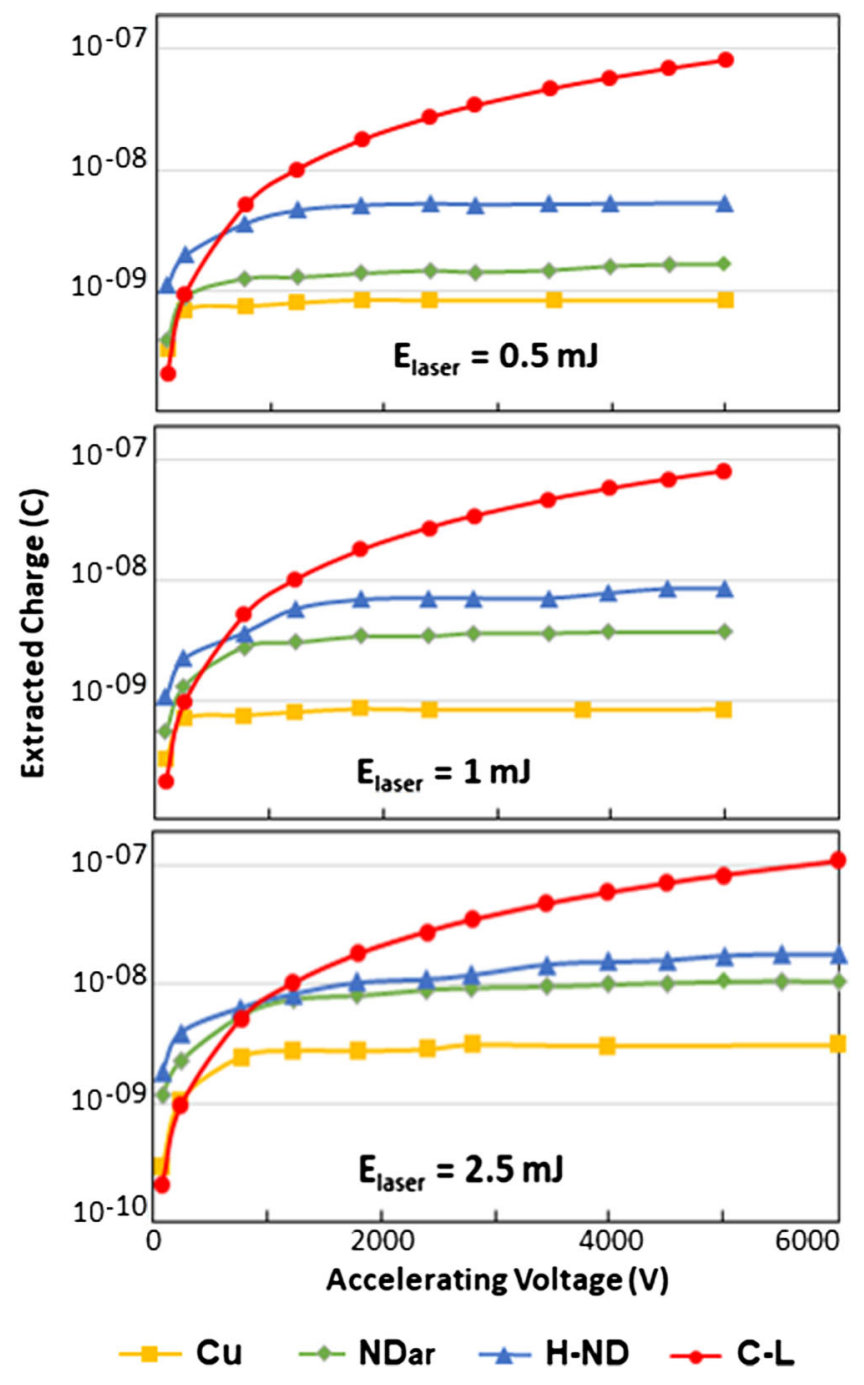

FIG. 5. Photoemitted extracted charge of the $\mathrm{Cu}, \mathrm{ND}_{\mathrm{ar}}$ and H-ND-based cathodes and the Child-Langmuir (C-L) red curve as a function of the accelerating voltage.

function of the accelerating voltage, at three different laser energy values. The red curve (circles) in each graphic represents the Child Langmuir (C-L) curve, which is a theoretical upper bound of current density $J$ allowed in the space-charge regime for a plane diode. The generic form of the $\mathrm{C}-\mathrm{L}$ equation is

$$
J=2.43 \times 10^{-6} \frac{V^{3 / 2}}{d^{2}},
$$

where $V$ is the accelerating voltage and $d$ is the anodecathode distance.

From the results shown in Fig. 5, it is seen that at low accelerating voltage, the charge extracted from the NDbased photocathodes exceeds the predictions of the ChildLangmuir law. This peculiar result is due to the plasma formation near the cathode surface $[33,36]$. Although the utilized laser energies are lower than the ablation threshold, 
TABLE I. Quantum efficiency (QE) values of the $\mathrm{Cu}, \mathrm{ND}_{\mathrm{ar}}$ and H-ND-based photocathodes at three different values of laser energy $\left(E_{\text {laser }}\right)$.

\begin{tabular}{lcccc}
\hline \hline $\begin{array}{l}E_{\text {laser }} \\
(\mathrm{mJ})\end{array}$ & $\begin{array}{c}\text { Intensity } \\
\left(\mathrm{J} / \mathrm{cm}^{2}\right)\end{array}$ & $\begin{array}{c}\mathrm{QE}_{\mathrm{Cu}} \\
\text { (electrons/ } \\
\text { photons) }\end{array}$ & $\begin{array}{c}\mathrm{QE}_{\mathrm{ND}_{\mathrm{ar}}} \\
\text { (electrons/ } \\
\text { photons) }\end{array}$ & $\begin{array}{c}\mathrm{QE}_{\mathrm{H}-\mathrm{ND}} \\
\text { (electrons/ } \\
\text { photons) }\end{array}$ \\
\hline 0.5 & $1.25 \times 10^{-3}$ & $4.20 \times 10^{-6}$ & $1.65 \times 10^{-5}$ & $5.00 \times 10^{-5}$ \\
1.0 & $2.50 \times 10^{-3}$ & $4.10 \times 10^{-6}$ & $1.70 \times 10^{-5}$ & $4.00 \times 10^{-5}$ \\
2.4 & $6.00 \times 10^{-3}$ & $4.30 \times 10^{-6}$ & $1.80 \times 10^{-5}$ & $3.70 \times 10^{-5}$ \\
\hline \hline
\end{tabular}

the emitted electrons can ionize the atoms of contaminants and adsorbates, forming a plasma layer on the diamond surface. This plasma layer thickens with increasing laser energy, decreasing the anode-cathode distance $d$ and thus modifying the current density values from the C-L equation (8).

At higher bias voltages, none of the photocathodes provide the level of charge predicted by C-L law. The charge from the $\mathrm{Cu}$ and $\mathrm{ND}_{\mathrm{ar}}$ cathodes saturates for each of the three laser energies at voltages higher than $2000 \mathrm{~V}$, however charge saturation of the H-ND cathode is achieved only at low laser energy of $0.5 \mathrm{~mJ}$ and not at 1 and $2.5 \mathrm{~mJ}$ (this statement is more evident when results are plotted on a linear scale).

The $\mathrm{QE}$ is calculated as the ratio of emitted electrons and incident photons. The QE values are reported in Table I.

The QE values of Table I are plotted in Fig. 6, to better represent the trend of the quantum efficiency of the three cathodes as a function of the laser energy.

As expected, the photocathodes based on $\mathrm{ND}_{\mathrm{ar}}$ and $\mathrm{H}-\mathrm{ND}$ are more efficient than that of the $\mathrm{Cu}$ one, used as reference. This high performance in the UV range is due to the good optical and electrical properties of the diamond [37-39]. Moreover, the graphite component of the ND

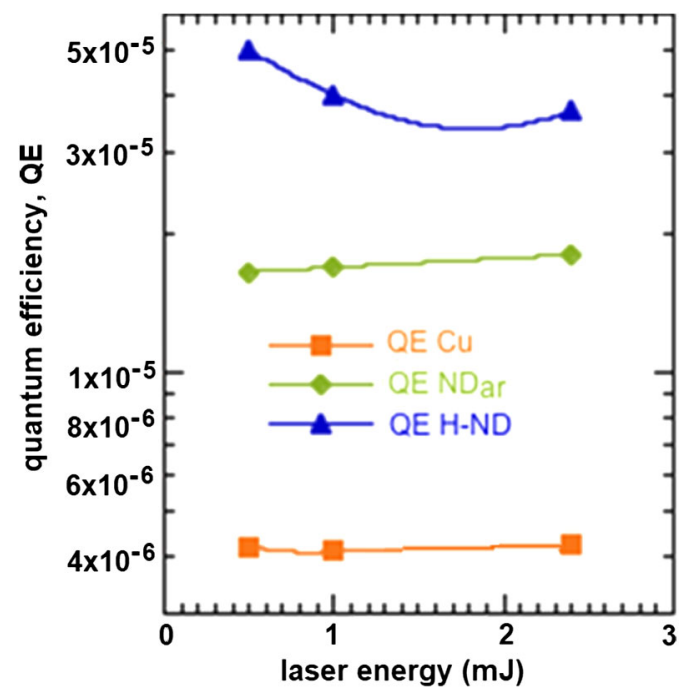

FIG. 6. Quantum efficiency (QE) of the $\mathrm{Cu}, \mathrm{ND}_{\text {ar }}$ and H-NDbased photocathodes vs laser energy. powder plays a fundamental role in the enhancement of the photoemission, as well shown in several previous works $[18,19,40,41]$. The highest QE found for the H-ND-based photocathode is due to the NEA character of its hydrogenated surface.

For the case of $\mathrm{Cu}$ and $\mathrm{ND}_{\mathrm{ar}}$ cathodes, the $\mathrm{QE}$ is constant as a function of increasing laser energy, consistent with the expected one-photon absorption process. But for the H-ND cathode, the QE decreases with increasing laser energy. As already noted, the extracted charge from the H-ND surface does not reach complete saturation. Thus, it would be necessary to increase the accelerating voltage to collect the maximum number of electrons.

Figure 7 shows the temporal response of the three cathodes. The laser signals (dashed yellow) show the typical bell-shaped curve with a FWHM about 20 ns that corresponds to the $\mathrm{KrF}$ pulse duration; the electron signals (dotted brown) show curves with different width for the three cathodes. The shape of these curves generally depends on the presence of defects and/or impurity states in the crystalline lattice, on the absorption/reflection mechanisms and on the multiphoton processes that occur

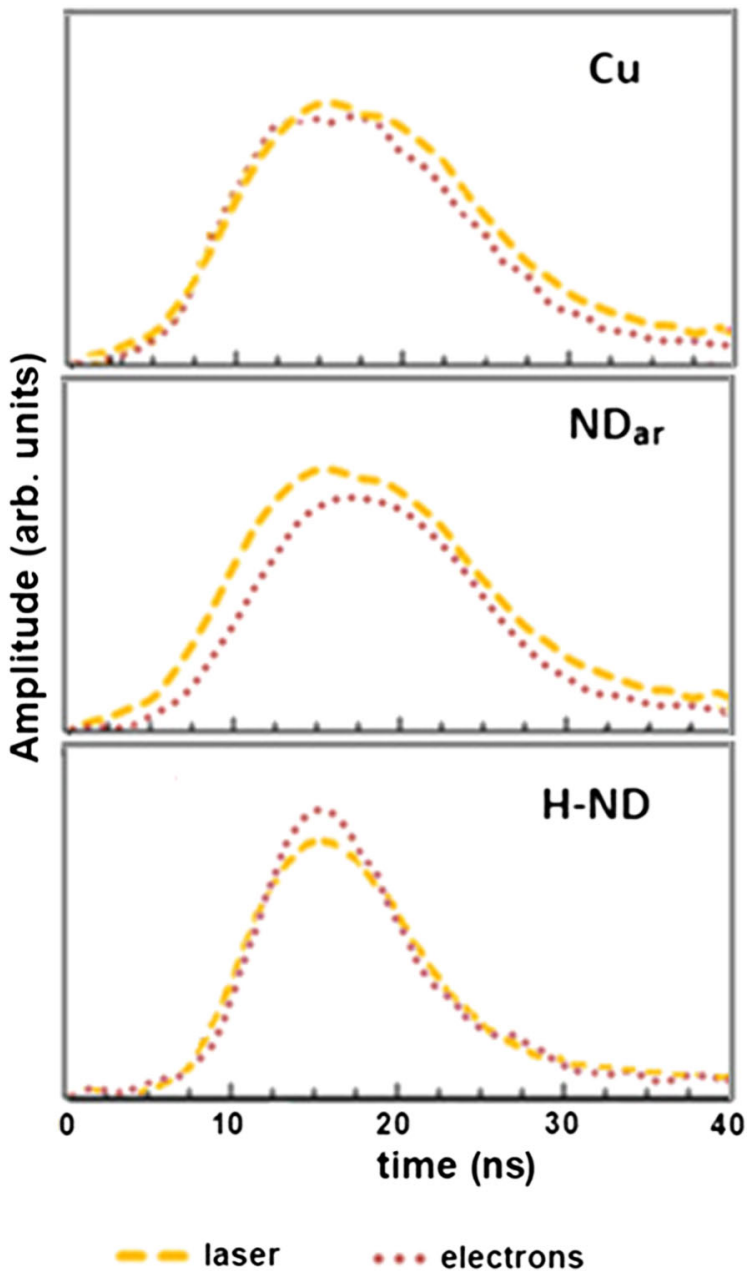

FIG. 7. Temporal response for the three photocathodes. 
during the laser-cathode interaction. A narrower curve means the contribution of the two-photon process $\left(J_{2}\right)$ is added to the one-photon process $\left(J_{1}\right)$, increasing the output and reducing the width of the total current signal $\left(J=J_{1}+J_{2}\right)$. This is observed for the $\mathrm{Cu}$ and $\mathrm{ND}_{\mathrm{ar}}$ photocathodes (the width at FWHM of electron signals is less than that of the corresponding laser pulse), whereas the H-ND cathode shows a wider electron signal compared with the laser pulse. From these data, we postulate that the NEA character of its surface diminishes the gap that the electrons have to overcome for their emission to vacuum, so the $J_{1}$ contribution is the highest for this PC.

Despite these considerations, the one-photon process remains the dominant mechanism for all the investigated $\mathrm{PCs}$, under the $\mathrm{KrF}$ irradiation at the wavelength of $248 \mathrm{~nm}$.

\section{CONCLUSIONS}

A comparative study of three photocathodes, two based on ND layers ( $\mathrm{ND}_{\mathrm{ar}}$ and $\left.\mathrm{H}-\mathrm{ND}\right)$ and $\mathrm{Cu}$ as reference, was carried out. The photoemission measurements showed that the ND-based photocathodes exhibit quantum efficiencies higher than that of $\mathrm{Cu}$. In particular, the highest photoemission was found in H-ND-based photocathode thanks to the NEA character of its hydrogenated surface. By the analysis of photocurrent signals, we postulate that the contribution of the one-photon process (under $\mathrm{KrF}$ irradiation) is higher for the H-ND because of the NEA character of its surface, which reduces the energy gap between the valence band maximum and the vacuum level.

The paper also shows how it is possible to measure directly the quantum efficiency of a photocathode, without the use of a calibrated NIST photodiode. In the experimental section, we showed how the shunt calibration assures the recording of signals with rise time of a few ns.

[1] L. Giannessi et al., Seeding experiments at SPARC, Nucl. Instrum. Methods Phys. Res., Sect. A 593, 132 (2008).

[2] S. H. Kong, J. Kinross-Wright, D. C. Nguyen, and R. L. Sheffield, Photocathodes for free electron lasers, Nucl. Instrum. Methods Phys. Res., Sect. A 358, 272 (1995).

[3] C. Bostedt et al., Experiments at FLASH, Nucl. Instrum. Methods Phys. Res., Sect. A 601, 108 (2009).

[4] A. Todd, State-of-the-art electron guns and injector designs for energy recovery linacs (ERL), Nucl. Instrum. Methods Phys. Res., Sect. A 557, 36 (2006).

[5] K. T. McDonald, Design of the laser-driven RF electron gun for the BNL accelerator test facility, IEEE Trans. Electron Devices 35, 2052 (1988).

[6] I. Ben-Zvi, in Proceedings of the 1991 Particle Accelerator Conference, San Francisco, CA, 1991 (IEEE, New York, 1991).

[7] D. T. Palmer et al., in Proceedings of the Particle Accelerator Conference, Vancouver, BC, Canada, 1997 (IEEE, New York, 1997).
[8] R. Xiang and J. Teichert, Photocathodes for high brightness photo injectors, Phys. Procedia 77, 58 (2015).

[9] L. Faillace, Recent advancements of RF guns, Phys. Procedia 52, 100 (2014).

[10] J. Scifo et al., Nano-machining, surface analysis and emittance measurements of a copper photocathode at SPARC_LAB, Nucl. Instrum. Methods Phys. Res., Sect. A 909, 233 (2018).

[11] A. Giribono et al., EuPRAXIA@ @PARC_LAB: The highbrightness RF photo-injector layout proposal, Nucl. Instrum. Methods Phys. Res., Sect. A 909, 282 (2018).

[12] J. Scifo et al., Nano-machining, surface analysis and emittance measurements of a copper photocathode at SPARC_LAB, Nucl. Instrum. Methods Phys. Res., Sect. A 909, 233 (2018).

[13] A. Braem, C. Joram, F. Piuz, E. Schyns, and J. Séguinot, Technology of photocathode production, Nucl. Instrum. Methods Phys. Res., Sect. A 502, 205 (2003).

[14] L. Cultrera, A. Pereira, C. Ristoscu, A. Clozza, F. Tazzioli, and C. Vicario, Pulsed laser deposition of Mg thin films on $\mathrm{Cu}$ substrates for photocathode applications, Appl. Surf. Sci. 248, 397 (2005).

[15] O. Siegmund, J. Vallerga, J. McPhate, J. Malloy, A. Tremsin, A. Martin, M. Ulmer, and B. Wessels, Development of GaN photocathodes for UV detectors, Nucl. Instrum. Methods Phys. Res., Sect. A 567, 89 (2006).

[16] C. Feng, Y. Zhang, J. Liu, Y. Qian, J. Zhang, J. Zhao, F. Shi, and X. Bai, Optimized chemical cleaning procedure for enhancing photoemission from GaAs photocathode, Mater. Sci. Semicond. Process. 91, 41 (2019).

[17] C. Hernandez-Garcia et al., Charge production studies from $\mathrm{Cs}_{2} \mathrm{Te}$ photocathodes in a normal conducting RF gun, Nucl. Instrum. Methods Phys. Res., Sect. A 871, 97 (2017).

[18] L. Velardi, A. Valentini, and G. Cicala, Highly efficient and stable ultraviolet photocathode based on nanodiamond particles, Appl. Phys. Lett. 108, 083503 (2016).

[19] L. Velardi, A. Valentini, and G. Cicala, UV photocathodes based on nanodiamond particles: Effect of carbon hybridization on the efficiency, Diam. Relat. Mater. 76, 1 (2017).

[20] J. Robertson and M. J. Rutter, Band diagram of diamond and diamond-like carbon surfaces, Diam. Relat. Mater. 7, 620 (1998).

[21] F. J. Himpsel, J. A. Knapp, J. A. VanVechten, and D. E. Eastman, Quantum photoyield of diamond(111) — A stable negative-affinity emitter, Phys. Rev. B 20, 624 (1979).

[22] J. B. Cui, J. Ristein, and L. Ley, Low-threshold electron emission from diamond, Phys. Rev. B 60, 16135 (1999).

[23] F. A. Almeida, E. Salgueiredo, F. J. Oliveira, R. F. Silva, D. L. Baptista, S. B. Peripolli, and C. A. Achete, Interfaces in nano-/microcrystalline multigrade CVD diamond coatings, ACS Appl. Mater. Interfaces 5, 11725 (2013).

[24] A. F. Sartori, S. Orlando, A. Bellucci, D. M. Trucchi, S. Abrahami, T. Boehme, T. Hantschel, W. Vandervorst, and J. G. Buijnsters, Laser-induced periodic surface structures (LIPSS) on heavily boron-doped diamond for electrode applications, ACS Appl. Mater. Interfaces 10, 43236 (2018).

[25] G. Cicala, R. Brescia, M. A. Nitti, A. Romeo, D. Altamura, C. Giannini, M. Capitelli, P. Spinelli, and S. Schutzmann, Study of polycrystalline diamond deposition by continuous 
and pulsed discharges, Surf. Coat. Technol. 204, 1884 (2010).

[26] G. Cicala, D. Monéger, D. Cornacchia, P. Pesce, V. Magaletti, G. Perna, V. Capozzi, and M. Tamborra, Toward smooth MWPECVD diamond films: Exploring the limits of the hydrogen percentage in $\mathrm{Ar} / \mathrm{H} 2 / \mathrm{CH} 4$ gas mixture, Surf. Coat. Technol. 211, 152 (2012).

[27] G. Cicala, A. Massaro, L. Velardi, G. S. Senesi, and A. Valentini, Self-assembled pillar-like structures in nanodiamond layers by pulsed spray technique, ACS Appl. Mater. Interfaces 6, 21101 (2014).

[28] G. Cicala, A. Massaro, L. Velardi, G. S. Senesi, G. Perna, D. Marzulli, D. Melisi, G. De Pascali, A. Valentini, and V. Capozzi, Enhancement of surface electrical current on silicon via nanodiamond particles deposited by pulsed spray technique, Phys. Status Solidi A 212, 2862 (2015).

[29] A. Valentini, D. Melisi, G. De Pascali, G. Cicala, L. Velardi, and A. Massaro, High-efficiency nanodiamondbased ultraviolet photocathodes, International Patent No. WO/2017/051318 (2017).

[30] http://www.diamondsandtools.com/.

[31] J. H Bechtel, W. Lee Smith, and N. Bloembergen, Twophoton photoemission from metals induced by picosecond laser pulses, Phys. Rev. B 15, 4557 (1977).

[32] J. Lin and T. F. George, Laser-generated electron emission from surfaces: Effect of the pulse shape on temperature and transient phenomena, J. Appl. Phys. 54, 382 (1983).

[33] F. Belloni, G. Caretto, A. Lorusso, V. Nassis, A. Perrone, and M. V. Siciliano, Photo-emission studies from $\mathrm{Zn}$ cathodes under plasma phase, Radiat. Eff. Defects Solids 160, 587 (2005).

[34] P. J. Olver, Introduction to Partial Differential Equations (Springer, New York, 2014), ISBN 978-3-319-02098-3.

[35] Q. Jiang and Z. Wen, Thermodynamics of Materials. Heat Capacity Entropy, and Nanothermodynamics (Springer, Berlin 2011), Chap. 3.

[36] G. Caretto, P. Miglietta, V. Nassisi, A. Perrone, and M. V. Siciliano, Photoelectron performance of Y thin films and Y smooth bulk cathodes, Radiat. Eff. Defects Solids 163, 365 (2008).

[37] D. Vouagner, Y. Show, B. Kiraly, B. Champagnon, and J. P. Girardeau-Montaut, Photoemission characteristics of diamond films, Appl. Surf. Sci. 168, 79 (2000).

[38] A. S. Tremsin and O. H. W. Siegmund, UV photoemission efficiency of polycrystalline CVD diamond films, Diam. Relat. Mater. 14, 48 (2005).

[39] G. Cicala, V. Magaletti, A. Valentini, M. A. Nitti, A. Bellucci, and D. M. Trucchi, Photo-and thermionic emission of MWPECVD nanocrystalline diamond films, Appl. Surf. Sci. 320, 798 (2014).

[40] W. Zhu, G. P. Kochanski, and S. Jin, Low-field electron emission from undoped nanostructured diamond, Science 282, 1471 (1998).

[41] J. B. Cui, M. Stammler, J. Ristein, and L. Ley, Role of hydrogen on field emission from chemical vapor deposited diamond and nanocrystalline diamond powder, J. Appl. Phys. 88, 3667 (2000). 